\title{
BMJ Open Acupuncture treatment for ischaemic stroke in young adults: protocol for a randomised, sham-controlled clinical
} trial

\author{
Lifang Chen, ${ }^{1}$ Jianqiao Fang, ${ }^{1,2}$ Xiaoming Jin, ${ }^{3}$ Crystal Lynn Keeler, ${ }^{2}$ Hong Gao, ${ }^{1}$ \\ Zhen Fang, ${ }^{4}$ Qin Chen ${ }^{1}$
}

To cite: Chen L, Fang J, Jin $\mathrm{X}$, et al. Acupuncture treatment for ischaemic stroke in young adults: protocol for a randomised, sham-controlled clinical trial. BMJ Open 2016;6:e010073. doi:10.1136/bmjopen-2015010073

- Prepublication history for this paper is available online. To view these files please visit the journal online (http://dx.doi.org/10.1136/ bmjopen-2015-010073).

Received 22 September 2015 Revised 18 November 2015 Accepted 7 December 2015

CrossMark

For numbered affiliations see end of article.

Correspondence to Professor Jianqiao Fang; fangjianqia07532@163.com

\begin{abstract}
Introduction: Stroke in young adults is not uncommon. Although the overall incidence of stroke has been recently declining, the incidence of stroke in young adults is increasing. Traditional vascular risk factors are the main cause of young ischaemic stroke. Acupuncture has been shown to benefit stroke rehabilitation and ameliorate the risk factors for stroke. The aims of this study were to determine whether acupuncture treatment will be effective in improving the activities of daily living (ADL), motor function and quality of life (QOL) in patients of young ischaemic stroke, and in preventing stroke recurrence by controlling blood pressure, lipids and body weight.
\end{abstract}

Methods and analysis: In this randomised, shamcontrolled, participant-blinded and assessor-blinded clinical trial, 120 patients between 18 and 45 years of age with a recent (within 1 month) ischaemic stroke will be randomised for an 8-week acupuncture or sham acupuncture treatment. The primary outcome will be the Barthel Index for ADL. The secondary outcomes will include the Fugl-Meyer Assessment for motor function; the World Health Organization Quality of Life BREF (WHOQOL-BREF) for QOL; and risk factors that are measured by ambulatory blood pressure, the fasting serum lipid, body mass index and waist circumference. Incidence of adverse events and longterm mortality and recurrence rate during a 10-year and 30-year follow-up will also be investigated. Ethics and dissemination: Ethics approval was obtained from the Ethics Committee of The Third Affiliated Hospital of Zhejiang Chinese Medical University. Protocol V.3 was approved in June 2013. The results will be disseminated in a peer-reviewed journal and presented at international congresses. The results will also be disseminated to patients by telephone during follow-up calls enquiring on the patient's post-study health status.

Trial registration number: ChiCTR-TRC- 13003317; Pre-results.

\section{INTRODUCTION}

Stroke is one of the most frequent causes of death and chronic disability in China. ${ }^{1}$ Although the overall incidence of stroke has

\section{Strengths and limitations of this study}

- This will be the first study protocol concerning the effect of acupuncture for treating and preventing stroke specifically in young adults.

- Methodological strengths included: rigorous randomised, sham-controlled, participants-blinded and assessors-blinded, and lengthy follow-ups.

- The sham acupuncture (superficial needle insertion and minimal stimulation at non-acupoint and non-meridian areas) may be associated with larger effects than pharmacological and other physical placebos, and produce some physiological activity.

been recently declining, the incidence of stroke in young adults is increasing. Approximately $10-14 \%$ of ischaemic strokes occur in adults between 18 and 45 years of age. ${ }^{2}{ }^{3}$ While a specific definition of 'young stroke' does not exist, the vast majority of authors consider 'young stroke' as strokes that occur in individuals under 45 years of age. ${ }^{4}$ For the purposes of this research, we defined 'young stroke' as strokes occurring in adults between 18 and 45 years of age and designed a randomised clinical trial to address the particular needs and differences of this younger age group.

The causes of young stroke are heterogeneous; however, traditional vascular risk factors, such as hypertension, hyperlipidaemia, obesity, smoking, alcohol drinking, and diabetes mellitus, are suggested to be the main risk factors. ${ }^{5}$ This finding challenges the traditional view that young stroke is often caused by 'rare' events. ${ }^{6}$ Stroke in the younger age group may have a dramatic impact on the quality of life (QOL) for survivors, given their longer life expectancy and life stage. This age group has a longer life expectancy than do older adults who suffer from strokes; also, these adults are likely to 
be wage-earning caregivers for either children and/or ageing parents. Disability from young stroke may create an additional economic burden on the family along with a societal impact. Owing to the potentially greater impact of disability-adjusted life years for this age group, more attention should be paid to the rehabilitation programme and prevention of recurrence of young stroke.

Our previous two separate clinical trials, one for subacute stroke and the other for acute stroke, have been investigating the impact of acupuncture on stroke outcomes overall. ${ }^{7} 8$ During this investigation, we have found that young adults with stroke may obtain 'De Qi' more easily and recover better than do older patients ('De Qi' is an acupuncture term for the sensation of 'qi' arriving on the needle, also known as 'obtaining qi', causing the acupuncture needle to elicit the patient's feeling of soreness, numbness, distension, heaviness or electric shock around the point together with the practitioner's feeling of tenseness around the needle). A literature search for 'acupuncture for young stroke' did not yield any results, while intervention outcomes may have a different impact on young stroke. To date, most of the literature that is focused on young stroke comprises observational studies and few intervention studies have been published. Randomised clinical trials that investigate the impact of acupuncture on young stroke are currently scarce. Therefore, the role of acupuncture in young stroke treatment deserved further study.

Acupuncture originated more than 3000 years ago in China and has gained popularity in the USA since the late 1970s. ${ }^{9}$ Over its 2500 years of development, a wealth of experience has accumulated in the practice of acupuncture for stroke treatment and prevention. According to a report by the WHO in 2002, 'strokes and their sequelae are another major indication for acupuncture. ${ }^{10}$ Furthermore, recent reviews have shown positive, but limited, effectiveness of acupuncture as an adjunct treatment to the conventional care of stroke. ${ }^{11-13}$ Acupuncture has also been reported to have benefits for treating risk factors for stroke, including hypertension, hyperlipidaemia and obesity. ${ }^{14-16}$ This intervention modality has a long history of safety and efficacy. As long as trained practitioners perform acupuncture using clean needle techniques, it is a generally safe procedure; ${ }^{17}$ however, some studies were at risk of bias. Future studies should focus on the appropriate modality of acupuncture intervention, sham controls, the appropriate length of treatment and the development of more precise tools to assess outcomes.

This clinical trial focuses on young ischaemic stroke. The study will test the hypotheses that acupuncture (when compared with a sham control group) will be effective in improving several outcome measures after a first-time young stroke, as well as improving risk factors that are associated with recurrent stroke. The outcome measures will include independence in activities of daily living (ADL), motor function and QOL. The risk factors that will be examined include blood pressure (BP), lipids and body weight.
METHODS

\section{Study design}

This is a 20-week, single-centre, randomised, shamcontrolled, participant-blinded and assessor-blinded, exploratory clinical trial. The study patient flow outline is shown in figure 1 and the trial schedule is shown in table 1. Protocol modifications are not expected. The eligibility criteria are fairly well established; however, if a trial modification is necessary, any change in the criteria or methodology will be communicated to the entire research team in a conference. All changes will be included in the final write-up for a journal submission.

\section{Study setting, recruitment and ethics}

This trial is registered at Chictr.org (number ChiCTR-TRC-13003317) and will be conducted in accordance with the Declaration of Helsinki. We will recruit participants by advertising in the local newspapers, health-related TV programmes, internet and WeChat in Hangzhou city. We will conduct this trial in the acupuncture and rehabilitation departments of a teaching hospital that is located in the centre of Hangzhou city. There are 50 beds in the inpatient clinic and 80 beds in the outpatient clinic of the acupuncture department, and 60 beds in the rehabilitation department. All patients will provide written informed consent. The research coordinator will arrange an in-person meeting with potential participants to discuss the study in detail (ie, the study purpose, procedures and time commitment, as well as the potential risks and benefits associated with participation in the study) and to obtain written informed consent. The confidentiality of participant records will be protected. At the time of enrolment, each patient will be assigned a unique study identification number, which is the only direct identifier included on all study paper forms and in the electronic database. During and after the trial, all records will remain secure, either in a locked filing cabinet in a secure area or in password-protected computer files. Compensation for trial participants for trial-related harms will be provided by The Third Affiliated Hospital of Zhejiang Chinese Medical University and will be made on a case-by-case basis. All hospital and medical care related to any possible harm from the trial will be provided free of charge to the patient.

\section{Patient population}

Patients between 18 and 45 years of age with a recent (within 1 month) ischaemic stroke are eligible for this study. Patients who will be included as eligible participants should display clear consciousness and stable vital signs, with a modified Rankin Score (mRS) 2-4, and have no aphasia or cognitive dysfunction.

The exclusion criteria will include patients with intracranial lesions or cerebrovascular disease history that have caused sequelae, such as movement disorders, mood disorders, cognitive dysfunction or speech problems; or individuals who are pregnant or breast 
Figure 1 Route diagram of study design (AG, acupuncture group; QOL, quality of life; SG, sham acupuncture group).

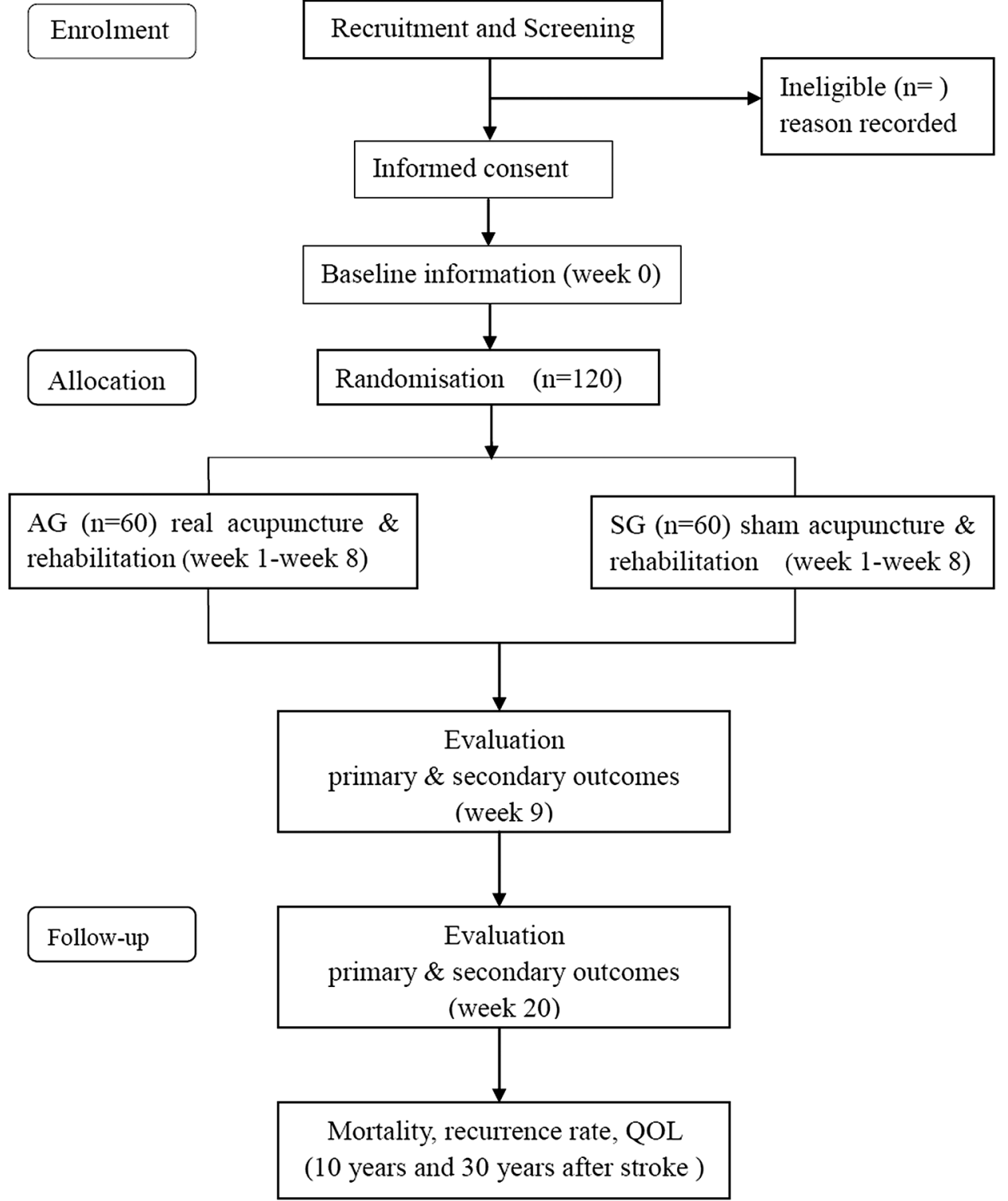

feeding. Patients with serious diseases that are related to the heart, liver, kidney or haematopoietic system will not be allowed to participate. Patients who are not interested in this study or are participating in other clinical trials will also be excluded. To increase the credibility of the sham control, patients with prior experience of acupuncture will be excluded from our final protocol. Since many people in China have previously received acupuncture, this exclusion criterion may prolong the study period by excluding otherwise eligible participants. Nevertheless, we believe that this exclusion category will improve the quality of the research by providing a more effective sham control. Changes that affect trial participants directly will be explained in person by their coordinators.

\section{Randomisation and blinding}

Randomisation will be computer generated by independent research staff using SAS 9.3 software (order number: 99TKBM). The generated list of random numbers will be placed into sequentially numbered, opaque, sealed envelopes. Consecutive patients will be randomly assigned to an acupuncture group (AG) or a sham acupuncture group (SG) in a 1:1 ratio according to the information received from the envelopes. Two acupuncture doctors will be designated to perform the acupuncture treatment for the AG or SG separately. The study coordinator, who will not be involved in treatment or assessment, will inform the participants that they will receive either a 'traditional Chinese acupuncture' (verum) or a 'less-painful acupuncture developed especially for this study' (sham). All rehabilitation therapists, outcome assessors and data analysts will be blinded to group assignments.

\section{Interventions and comparison}

Both groups will receive conventional stroke rehabilitation treatment and care during the whole 20-week study 
Table 1 Trial schedule

\begin{tabular}{|c|c|c|c|c|c|c|}
\hline Assessment & Screening & $\begin{array}{l}\text { Baseline } \\
\text { Week } 0\end{array}$ & $\begin{array}{l}\text { Post-treatment } \\
\text { Week } 8\end{array}$ & $\begin{array}{l}\text { Follow-up } \\
\text { Week } 20 \\
\end{array}$ & $\begin{array}{l}\text { Follow-up } \\
\text { Year } 10\end{array}$ & $\begin{array}{l}\text { Follow-up } \\
\text { Year } 30 \\
\end{array}$ \\
\hline Informed consent & 0 & 0 & & & & \\
\hline Demographics & 0 & 0 & & & & \\
\hline Stroke type & 0 & 0 & & & & \\
\hline History of stroke & 0 & 0 & & & 0 & 0 \\
\hline History of disease & $\triangle$ & 0 & & & 0 & 0 \\
\hline Concomitant medication & $\bar{\triangle}$ & 0 & 0 & 0 & 0 & 0 \\
\hline Blood pressure & $\triangle$ & 0 & 0 & 0 & $\triangle$ & $\triangle$ \\
\hline Blood laboratory test & & 0 & 0 & 0 & $\triangle$ & $\triangle$ \\
\hline Body mass index & $\triangle$ & 0 & 0 & 0 & $\triangle$ & $\triangle$ \\
\hline Waist circumference & $\vec{\triangle}$ & 0 & 0 & 0 & $\bar{\triangle}$ & $\triangle$ \\
\hline Barthel Index & & 0 & 0 & 0 & 0 & 0 \\
\hline FMA Motor Scale & & 0 & 0 & 0 & $\triangle$ & $\triangle$ \\
\hline WHOQOL-BREF & & 0 & 0 & 0 & 0 & 0 \\
\hline Adverse events & & & 0 & 0 & & \\
\hline Mortality & & & & & 0 & 0 \\
\hline Recurrence of stroke & & & & & 0 & 0 \\
\hline Treatment method & & & & & 0 & 0 \\
\hline
\end{tabular}

period. The programme was designed according to the Chinese stroke rehabilitation treatment guidelines and will be consistent across groups, ${ }^{18}$ which included $2 \mathrm{~h}$ of physical therapy and one half-hour of occupational therapy (OT) for 5 days a week. Western medicine will be permitted for conventional symptomatic treatment (eg, antihypertensive drugs and lipid-lowering drugs). Chinese herbal medicine and Chinese patent drugs will be prohibited during the trial. The acupuncture intervention and comparison adheres to the Standards for Reporting Interventions in Controlled Trials of Acupuncture (STRICTA) guidelines. Two certified acupuncturists with more than 5 years of clinical experience will separately perform the Traditional Chinese Medicine (TCM) acupuncture or sham acupuncture for assigned patients. The $30 \mathrm{~min}$ acupuncture or sham treatment will be manipulated by manual stimulation and conducted at the bedside, in the supine position, three times per week (on Monday, Wednesday and Friday) for 8 weeks, and with no individual differences. For improving and monitoring adherence, every treatment form and evaluation form will be completed and signed by participants and researchers. Those who have completed all treatments and evaluations will receive some form of financial compensation.

\section{Acupuncture group}

For scalp acupuncture, filiform stainless steel needles (size $0.25 \mathrm{~mm} \times 40 \mathrm{~mm}$, Huatuo brand, manufactured by Suzhou Medical Appliance in Suzhou, Jiangsu Province China) will be inserted with the whole body of the needle through the motor area and the sensory area of the lesion side. The intervention will include ipsilateral body acupuncture points for the affected side, LI15 (Jianyu), LI11 (Quchi), LI10 (Shousanli), SJ6 (Zhigou) and LI4 (Hegu) for the upper extremities; bilateral points for the lower limbs, ST31 (Biguan), GB34 (Yanglingquan), SP10 (Xuehai), ST36 (Zusanli), ST40 (Fenglong), SP6 (Sanyinjiao) and LR3 (Taichong); and abdominal points, CV12 (Zhongwan), GV10 (Xiawan), CV6 (Qihai), CV4 (Guanyuan), ST25 (Tianshu) and ST15 (Daheng). The depth of insertion varies from 20 to $35 \mathrm{~mm}$, according to different points and the response of the patients to 'De Qi'.

\section{Sham acupuncture group}

Sham acupuncture in this trial is defined as acupuncture with superficial needle insertion and minimal stimulation at the non-acupoint and non-meridian areas. All needles for the sham group will be inserted superficially $(1-3 \mathrm{~mm})$ and without stimulation, to avoid 'De Qi'. ${ }^{19}$ The number of needles inserted, needle retention time and the frequency of treatment are all the same as in the AG. The locations and manipulations of the real AG and SG are detailed in table 2.

\section{Outcome measures}

The primary outcome

The primary outcome will be the Barthel Index (BI) for independence in ADL. Since there are several scoring guidelines for the $\mathrm{BI}$, we adopted the version of Mahoney and Barthel. ${ }^{20}$

\section{Secondary outcomes}

Secondary outcomes will include the Fugl-Meyer Assessment (FMA) Motor Scale, the World Health Organization Quality of Life BREF (WHOQOL-BREF), ambulatory BP, fasting serum lipid, body mass index (BMI) and waist circumference (WC). The FMA Motor Scale is highly recommended as a clinical and research 


LI15 (Jian yu) In the depression distal and anterior to the acromion, between the clavicular and acromial portions of the deltoid

LI11 (Qu chi) With the elbow flexed, on the lateral end of the elbow crease, in a depression between the end of the crease and the With the elbow flexed, on the lateral end of the elbow crease, in a depression
lateral epicondyle of the humerus, on the extensor carpi radialis longus muscle

LI10 (Shou

san li)

SJ6 (Zhi gou) 3 cun proximal to the dorsal wrist joint space ('dorsal wrist crease'), in a depression between the radius and the ulna,

LI4 (He gu)

2 cun distal to LI11, on the extensor carpi radialis longus muscle radial to the tendon of the extensor digitorum communis muscle

On the radial aspect of the hand, between the 1 st and 2nd metacarpal bones, closer to the 2nd metacarpal bone and approximately at its midpoint

Inferior to the anterior superior iliac spine and lateral to the sartorius muscle, at the level of the lower border of the pubic symphysis

GB34 (Yang In the depression anterior and inferior to the head of the fibula, between the peroneus longus and extensor digitorum ling quan) longus muscles

SP10 (Xue hai) With the knee flexed, 2 cun proximal and slightly medial to the medial superior border of the patella, in a depression on the vastus medialis muscle

ST36 (Zu san li) 3 cun distal to ST-35 ('lateral eye of the knee') and 1 fingerbreadth lateral to the anterior crest of the tibia, on the

\section{tibialis anterior muscle}

ST40 (Feng At the midpoint of the line joining ST-35 and ST-41, 2 fingerbreadths lateral to the anterior crest of the tibia

long)

SP6 (San yin

jiao) 3 cun proximal to the highest prominence of the medial malleolus, on the posterior border of the medial crest of the tibia

LR3 (Tai chong) On the dorsum of the foot, between the 1st and 2nd metatarsal bones, in the depression proximal to the

OR3 (Tai chong) On the dorsum of the foot, between the 1st and 2nd metatarsal bones, in the
metatarsophalangeal joints and the proximal angle between the two bones

CV12 (Zhong On the anterior midline, 4 cun superior to the umbilicus

wan)

CV10 (Xia wan) On the anterior midline, 2 cun superior to the centre of the umbilicus

CV6 (Qi hai) On the anterior midline, 1.5 cun inferior to the umbilicus

CV4 (Guan On the anterior midline, 3 cun inferior to the umbilicus

yuan)

ST25 (Tian shu) 2 cun lateral to the umbilicus

ST15 (Da heng) 4 cun lateral to the centre of the umbilicus, on the mamillary line

The motor area $0.5 \mathrm{~cm}$ posterior to the midpoint of the anteroposterior line defines the upper limit of the motor area. The lower limit

intersects the eyebrow-occiput line at the anterior border of the natural hairline on the temple

The sensory A line parallel to the motor area and $1.5 \mathrm{~cm}$ behind it

area

${ }^{*}$ Manipulations of real acupuncture. For scalp acupuncture, the needle is swiftly inserted into the subcutaneous tissue of the scalp in a horizontal direction. When the tip of the needle reaches the subgaleal layer and the practitioner feels low insertion resistance, the needle is further inserted to a depth of $30-40 \mathrm{~mm}$ by the twirling method. Three needles are used for each area. For body acupuncture, the needle is inserted into the points to a depth of between 30 and $40 \mathrm{~mm}$ according to different regions. Manual stimulation will be applied to the needles until the patients experience the needling sensation (called 'Deqi' in Chinese acupuncture). For electroacupuncture of CV12 (Zhong wan) and CV10 (Xia wan), ST25 (Tianshu, 2 sides) points, SDZ-? B Nerve

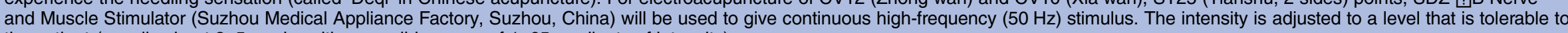
the patient (usually about 3-5 grade, with a possible scope of $1-65$ gradients of intensity).

†Manipulations of sham acupuncture. The same stainless needles $(0.25 \mathrm{~mm} \times 40 \mathrm{~mm}$, described above) will be used. For body acupuncture, 20 needles will be inserted $1 \mathrm{~cm}$ lateral away from the actual acupoints; and the same electroacupuncture instrument will be used, but only with 1 grade of intensity. For scalp acupuncture, needles will be inserted $1 \mathrm{~cm}$ anterior away from the motor area and the sensory area of the lesion side.

†Manipulations of two groups. The acupuncturists of two groups will insert needles in about 5-7 min, during which time minimal interaction with the patients will be made. Then the patients are

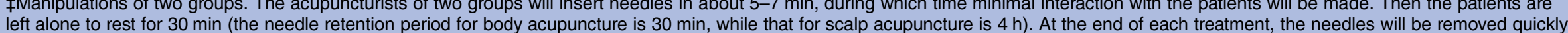

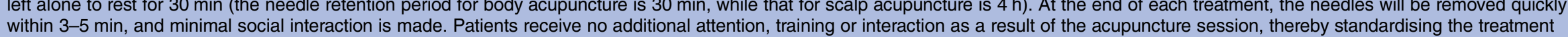
and control groups. 
tool for evaluating changes in motor impairment following stroke. ${ }^{21}$ The WHOQOL-BREF is a 26-item version of the WHOQOL-100 assessment that was developed in the context of four domains of QOL and is a sound, cross-culturally valid assessment of QOL. ${ }^{22}$ We will use the official Chinese version of the WHOQOL-BREF, which has been approved by the WHOQOL Group. ${ }^{23}$ $\mathrm{BP}$, lipid, BMI and WC are risk factors for recurrent stroke and represent relevant secondary outcome measurements. Patients will receive ambulatory BP measurements, and the average systolic and diastolic BP levels will be compared. The fasting serum lipid test will include total cholesterol, low-density lipoprotein cholesterol, high-density lipoprotein cholesterol and triglycerides. The BMI will be derived from the mass (weight) and height of an individual with the value universally being expressed in units of $\mathrm{kg} / \mathrm{m}^{2}$. The WC will be measured by starting from the top of the hipbone and measuring around the waist at the level of the umbilicus. All primary and secondary outcomes will be measured at weeks 0,8 and 20. To observe the long-term preventive effect and prognosis, we will follow-up with all participants annually through telephone contact, home visits or clinic visits to collect data on mortality, recurrence rate of stroke, $\mathrm{ADL}$ and QOL at 10-year and 30-year increments after stroke.

\section{Incidence of adverse events}

All participants will be requested to voluntarily report information about adverse events (AEs) at each visit. AEs that occur during the trial will be recorded and assessed by the investigators. Acupuncture-related AEs might include local bleeding, haematoma, pallor, sweating or dizziness, fainting during acupuncture treatment, unbearable prickling or retained needle after treatment.

\section{Data collection, management and monitoring}

To promote participant retention and complete follow-up, all interventions and outcome measurements are free to participants, and financial incentives will be provided to those who completed all interventions and/ or follow-ups. Participants who discontinue interventions but do not drop out will be invited to enter the follow-up phase and will receive assessments throughout the remainder of the study period.

The Case Report Form (CRF), Treatment Form and Adverse Events Form will be first completed on paper copies and then double entered into the Electronic Data Capture (EDC) system electronically by two independent investigators to act as the first level of control to ensure the accuracy of the data. The second level of data integrity will include data monitoring and validation that will be conducted on a regular basis throughout the study. The original CRFs and all other forms (including the consent forms) will be archived securely at The Third Affiliated Hospital of Zhejiang Chinese Medical University for 5 years following publication of the last paper or report from the study.
The safety of the study will be monitored by a Data and Safety Monitoring Board (DSMB) of the Clinical Evaluation Center of Zhejiang Provincial Hospital of TCM, which consists of independent clinical experts and statisticians with access to unblinded data. The DSMB is independent from the sponsor, the competing interests and the investigational site and will review the performance and safety of the trial monthly.

The criteria for unblinding and discontinuing allocated interventions for a given trial participant include having a recurrent stroke, having serious complications of stroke or experiencing serious acupuncture-related AEs (if any), which have been described previously. The DSMB will reveal a participant's allocated intervention, and make the final decision to terminate the trial.

The final trial data set will be under the custody of The Third Affiliated Hospital of Zhejiang Chinese Medical University. The data manager from the Clinical Evaluation Center of Zhejiang Provincial Hospital of TCM will have access to the complete, anonymous final data set. Access to the final data set or identifiable data by others will require written requests to be approved by the DSMB of the Clinical Evaluation Center of Zhejiang Provincial Hospital of TCM, The Third Affiliated Hospital of Zhejiang Chinese Medical University and all study investigators.

\section{Sample size estimates}

The sample size was determined using the results of our previous clinical trial. ${ }^{24}$ We expected that the primary efficacy parameter (BI) of the AG would be 10 values higher than that of the SG and that the SD would be approximately 18. A two-sided 5\% significance level and $80 \%$ power were considered, and the following equation was used:

$$
\mathrm{n}=\frac{2\left(\left(\mathrm{z}_{\mathrm{a}} / 2\right)+\mathrm{z}_{\beta}\right)^{2} \sigma^{2}}{\Delta^{2}}
$$

On the basis of the calculation, approximately 51 participants in each group would be required to have a sufficient sample size. With an estimated dropout rate of $15 \%$, each group required 60 initial participants.

\section{Statistical analysis}

Analyses will be conducted at the Clinical Research Institute of Zhejiang Provincial Hospital of TCM by blinded biostatisticians. SAS software, V.9.3, will be used for all analysis and a two-sided $\mathrm{p}<0.05$ will be considered statistically significant for all analyses. The full analysis set and per protocol set will both be conducted. Efficacy and safety analyses will be based on the intention-to-treat principle. Missing values will be handled by the mixed model for repeated measurements. Continuous variables with normal distribution will be expressed as the means with SDs and compared by an independent sample Student $t$ test. For abnormally distributed variables, the data will be expressed as medians with ranges and non- 
parametric tests will be used. Categorical variables will be expressed as number (\%) and analysed by $\chi^{2}$ test or Fisher's exact test.

Descriptive statistics will be used to detail baseline characteristics of patients, such as gender, type of stroke and risk factors, and will be compared between two groups using an independent sample Student $\mathrm{t}$ test or $\chi^{2}$ test. Repeated measures analysis of variance (ANOVA) will be used to analyse value changes of BI, FMA, WHOQOL-BREF, BP, blood lipid, BMI and WC across three testing time points (weeks 0,8 and 20). To detail all outcomes, an independent sample Student t test will be used to compare the final value changes (weeks 20-0) between the two groups of the above variables. The mortality and occurrence of stroke (including ischaemic stroke and cerebral haemorrhage) at the 10-year and 30-year follow-up will be analysed using the $\chi^{2}$ tests and log-rank test with the Kaplan-Meier survival curve analysis. Differences between the groups in causes of deaths will also be compared by the $\chi^{2}$ test. Safety analyses will be compared with the incidence of AEs in two groups using the $\chi^{2}$ test.

\section{DISCUSSION}

Most young adults with stroke have a financial need to return to work and a desire to enjoy an active social life. To improve the fine motor control poststroke and obtain more independence in ADL will require a higher level of recovery. Therefore, any treatments that contribute to functional rehabilitation should not be ignored, including traditional acupuncture. Despite the controversy in recent years concerning the role of acupuncture as a routine treatment for stroke, acupuncture may help to improve neurological deficits without important side effects, ${ }^{25-27}$ and is well accepted as part of comprehensive rehabilitation strategies in China. ${ }^{1}$ Prevention of ischaemic stroke recurrence in young patients also remains an important clinical problem. Acupuncture may not only be helpful in the treatment of poststroke rehabilitation without significant side effects but may also have the effect of regulating BP and lipid counts, as well as facilitate losing weight at the same time. ${ }^{12}{ }^{13}$ The acupuncture points used in this trial were designed for treating stroke and risk factors, which were selected in compliance with traditional Chinese medical principles and confirmed by modern research studies. ${ }^{28}$ Moreover, since acupuncture reactions and ' $\mathrm{De} \mathrm{Qi}$ ' are much easier to achieve in young adults, acupuncture treatment in these patients is expected to achieve better therapeutic effects. ${ }^{29}$

This trial focuses on ADL as the primary outcome because the ultimate goal of stroke rehabilitation is to improve the ADL of patients towards greater independence. Motor dysfunction is the most important sequela after stroke and has a significant impact on ADL measurements. Since young adults have a longer expected lifespan after the incidence of stroke, the QOL should also be considered. As a result, FMA and WHO-QOL were chosen as two secondary outcomes of treatment efficacy. Young patients have a much longer exposure to risk of a recurrent vascular event than do older patients, based on their age alone. Therefore, the study also examines risk-factor-related outcomes and the prevention of recurrent stroke by employing acupuncture for controlling hypertension, hyperlipidaemia, excessive weight and abdominal obesity. In addition, AEs and long-term outcomes with respect to recurrent events and mortality will be observed. In summary, the comprehensive rehabilitation and efficient secondary prevention of stroke in young individuals may greatly increase the number of productive years with good QOL. To the best of our knowledge, this will be the first trial of a rigorous, randomised, shamcontrolled trial of acupuncture that was designed specifically for treating and preventing young stroke.

There is a limitation in our trial that should be acknowledged. Including a third 'no acupuncture' group will improve the control design in our acupuncture trial. The possibility of a placebo effect, particularly for those who believe in traditional medicine, should not be dismissed; however, sham acupuncture interventions might, on average, be associated with larger effects than pharmacological and other physical placebos. ${ }^{30}$ Some of the sham acupuncture methods may also produce physiological activity. ${ }^{31}$ The nature of the placebo effect that is associated with sham acupuncture is an entire field of study unto itself. We consulted several Chinese acupuncture experts, many of whom believed that the effect of acupuncture for stroke is closely related with the stimulation of acupoints and meridians and 'De Qi,' which is in accordance with the ancient TCM theory that states, 'no De Qi, no effects.' These experts believe that sham acupuncture that is designed as 'superficial needle insertion and minimal stimulation at non-acupoint and non-meridian areas' in this trial could produce a placebo effect, but with no additional efficacy (or that a reduced influence by the sham acupuncture could be ignored when compared with real acupuncture). Nevertheless, in an acupuncture analgesia clinical trial, a sham acupuncture (of any type) could produce additional influence beyond that of a placebo. In our experience, sham acupuncture may produce different effects in different diseases. This may be because of the different mechanisms of acupuncture treatment for different diseases. For example, stroke is a major and complex disease while acupuncture treatment for stroke should focus on regulating the whole body and under the guideline of TCM theory. For painful syndromes, acupuncture treatment is much simpler to perform and easier to produce effects even if we do not select acupoints and meridians and only use local points. In this respect, the influence of sham acupuncture for pain may be greater than that for stroke. Taking into account that sham acupuncture may have little effect on the treatment of stroke, but could eliminate the placebo effect, we reasoned that sham acupuncture is an ideal control setting in this trial so long as it is well conducted. This conclusion is based on our clinical practice and requires further study. We will consider the 
nature of sham acupuncture in future studies to maximise the avoidance of this limitation.

\section{CONCLUSION}

Stroke in young adults requires a higher level of recovery and an effective prevention of recurrence. This study will be the first rigorous, randomised, sham-controlled trial that focuses on using acupuncture for treating and preventing young ischaemic stroke.

\section{Author affiliations}

${ }^{1}$ Department of Acupuncture and Moxibustion, The Third Affiliated Hospital of Zhejiang Chinese Medical University, Hangzhou City, Zhejiang Province, China ${ }^{2}$ Zhejiang Chinese Medical University, Hangzhou City, Zhejiang Province, China

${ }^{3}$ Department of Anatomy and Cell Biology \& Department of Neurosurgery, Indiana University School of Medicine, Indianapolis, Indiana, USA ${ }^{4}$ Department of Rehabilitation, The Third Affiliated Hospital of Zhejiang Chinese Medical University, Hangzhou City, Zhejiang Province, China

Acknowledgements The authors express their profound appreciation to all coordinators, therapists and evaluators for their diligence. They thank all of the stroke survivors and their caregivers who participated in this study.

Contributors LC, JF and XJ designed or conceptualised the trial, drafted and revised the manuscript. CLK, HG, ZF and QC designed and conducted the trial. All the authors have read, revised and approved this version of the manuscript.

Funding This work was supported by the programme of the National Key Discipline of Traditional Chinese Medicine-Acupuncture (number [2009]30), and partly funded by the International Exchange Program of Clinical Technology from Health and Family Planning Commission of Zhejiang Province (number [2014]48) and the Provincial Administration of Traditional Chinese Medicine of Zhejiang (number 2011ZGG003). The trial sponsor is The Third Affiliated Hospital of Zhejiang Chinese Medical University (219 Moganshan Road, Xihu District, Hangzhou City, Zhejiang Province 310005, China, 86-571-88393534).

Disclaimer The sponsor will have no role in the design and conduct of the study.

Competing interests None declared.

Patient consent Obtained.

Ethics approval The ethics committee of The Third Affiliated Hospital of Zhejiang Chinese Medical University approved the study (number ZSLL-KY-2013-016).

Provenance and peer review Not commissioned; externally peer reviewed.

Data sharing statement The results of our original research will be disseminated in a peer-reviewed journal and presented at international congresses.

Open Access This is an Open Access article distributed in accordance with the Creative Commons Attribution Non Commercial (CC BY-NC 4.0) license, which permits others to distribute, remix, adapt, build upon this work noncommercially, and license their derivative works on different terms, provided the original work is properly cited and the use is non-commercial. See: http:// creativecommons.org/licenses/by-nc/4.0/

\section{REFERENCES}

1. Liu M, Wu B, Wang WZ, et al. Stroke in China: epidemiology, prevention, and management strategies. Lancet Neurol 2007;6:456-64.

2. Ji R, Schwamm LH, Pervez MA, et al. Ischemic stroke and transient ischemic attack in young adults: risk factors, diagnostic yield, neuroimaging, and thrombolysis. JAMA Neurol 2013;70:51-7.

3. Kissela BM, Khoury JC, Alwell K, et al. Age at stroke: temporal trends in stroke incidence in a large, biracial population. Neurology 2012;79:1781-7.
4. Griffiths D, Sturm J. Epidemiology and etiology of young stroke. Stroke Res Treat 2011:2011:209370.

5. Bi Q, Wang L, Li X, et al. Risk factors and treatment of stroke in Chinese young adults. Neurol Res 2010;32:366-70.

6. Ferro JM, Massaro AR, Mas JL. Aetiological diagnosis of ischaemic stroke in young adults. Lancet Neurol 2010;9:1085-96.

7. Fang J, Chen L, Chen L, et al. Integrative medicine for subacute stroke rehabilitation: a study protocol for a multicentre, randomised, controlled trial. BMJ Open 2014;4:e007080.

8. Chen L, Fang J, Ma R, et al. Acupuncture for acute stroke: study protocol for a multicentre, randomised, controlled trial. Trials 2014;15:214.

9. Ernst E. Acupuncture-a critical analysis. J Intern Med 2006;259:125-37.

10. World Health Organization. Acupuncture: review and analysis of reports on controlled clinical trials. Geneva, Switzerland, 2002.

11. Vados L, Ferreira A, Zhao SF, et al. Effectiveness of acupuncture combined with rehabilitation for treatment of acute or subacute stroke: a systematic review. Acupunct Med 2015;33:180-7.

12. Lee SJ, Shin BC, Lee MS, et al. Scalp acupuncture for stroke recovery: a systematic review and meta-analysis of randomized controlled trials. Eur J Integr Med 2013;2:87-99.

13. Zhang J, Wang D, Liu M. Overview of systematic reviews and meta-analyses of acupuncture for stroke. Neuroepidemiology 2014;42:50-8

14. Flachskampf FA, Gallasch J, Gefeller O, et al. Randomized trial of acupuncture to lower blood pressure. Circulation 2007;115:3121-29.

15. Sui $\mathrm{Y}$, Zhao HL, Wong VC, et al. A systematic review on use of Chinese medicine and acupuncture for treatment of obesity. Obes Rev 2012;13:409-30.

16. Tuğrul Cabıoğlu M, Ergene N. Electroacupuncture therapy for weight loss reduces serum total cholesterol, triglycerides, and LDL cholesterol levels in obese women. Am J Chin Med 2005;33:525-33.

17. Lao L, Hamilton GR, Fu J, et al. Is acupuncture safe? A systematic review of case reports. Altern Ther Health Med 2003;1:72-83.

18. Zhang T. Chinese stroke rehabilitation treatment guidelines 2011 Chin J Rehabil Theory Pract 2012;18:301-18.

19. MacPherson $\mathrm{H}$, Asghar A. Acupuncture needle sensations associated with De Qi: a classification based on experts' ratings. J Altern Complement Med 2006;12:633-7.

20. Mahoney FI, Barthel DW. Functional evaluation: the Barthel Index. Md State Med J 1965;14:61-5.

21. Gladstone DJ, Danells CJ, Black SE. The Fugl-Meyer assessment of motor recovery after stroke: a critical review of its measurement properties. Neurorehabil Neural Repair 2002;16:232-40.

22. Skevington SM, Lotfy M, O'Connell KA, et al. The World Health Organization's WHOQOL-BREF quality of life assessment: psychometric properties and results of the international field trial. A report from the WHOQOL group. Qual Life Res 2004;13:299-310.

23. Wang X, Gao L, Zhang H, et al. Post-earthquake quality of life and psychological well-being: longitudinal evaluation in a rural community sample in northern China. Psychiatry Clin Neurosci 2000;54:427-33.

24. Chen LF, Fang JQ, Wu YY, et al. Acupuncture for motor dysfunction after subacute stroke: a multi-center randomized controlled trial. Chin Acupunct Moxibustion 2014;4:313-18.

25. Wu P, Mills E, Moher D, et al. Acupuncture in poststroke rehabilitation: a systematic review and meta-analysis of randomized trials. Stroke 2010;41:e171-9.

26. Wong AM, Su TY, Tang FT, et al. Clinical trial of electrical acupuncture on hemiplegic stroke patients. Am J Phys Med Rehabil 1999;78:117-22.

27. Uchida S, Hotta $\mathrm{H}$. Acupuncture affects regional blood flow in various organs. Evid Based Complement Altern Med 2008:5:145-51.

28. Naeser MA, Alexander MP, Stiassny-Eder D, et al. Real versus sham acupuncture in the treatment of paralysis in acute stroke patients: a CT scan lesion site study. Neurorehabil Neural Repair 1992;6:163-74.

29. Park SU, Ko CN, Bae HS, et al. Short-term reactions to acupuncture treatment and adverse events following acupuncture: a cross-sectional survey of patient reports in Korea. $J$ Altern Complement Med 2009;15:1275-83.

30. Linde K, Niemann K, Meissner K. Are sham acupuncture interventions more effective than (other) placebos? A re-analysis of data from the Cochrane review on placebo effects. Forsch Komplementmed 2010;5:259-64.

31. Carneiro M, Kawakita K. Re-analysis of acupuncture trials with sham interventions based on data from the Cochrane Review. Japanese Acupuncture Moxibustion 2015;1:1-11. 